nephron

Practice
Nephron 2018;138:119-128

DOI: $10.1159 / 000482016$
Received: June 26, 2017

Accepted: October 5, 2017

Published online: November 9, 2017

\title{
Value of Intraoperative Parathyroid Hormone Assay during Parathyroidectomy in Dialysis and Renal Transplant Patients with Secondary and Tertiary Hyperparathyroidism
}

\author{
Amr El-Husseini ${ }^{a, c}$ Kevin Wang ${ }^{a}$ Annick Edon ${ }^{a}$ David Saxon ${ }^{a}$ \\ Florence Lima $^{\mathrm{a}}$ David Sloan $^{\mathrm{b}}$ B. Peter Sawaya ${ }^{\mathrm{a}}$ \\ ${ }^{a}$ Division of Nephrology, Bone and Mineral Metabolism, University of Kentucky, Lexington, KY, USA; ${ }^{b}$ Section of \\ Endocrine Surgery, University of Kentucky, Lexington, KY, USA; ' Division of Nephrology, Mansoura University, \\ Mansoura, Egypt
}

\section{Keywords \\ Hyperparathyroidism · Intraoperative parathyroid hormone - Parathyroidectomy - End-stage renal disease . \\ Dialysis · Renal transplant}

\begin{abstract}
Background: In dialysis and renal transplant patients with secondary and tertiary hyperparathyroidism (HPT), the value of intraoperative parathyroid hormone (ioPTH) during parathyroidectomy (PTX) and its association with long-term PTH levels are unknown. The present study aims at evaluating the relationship of ioPTH with long-term PTH levels post-PTX in dialysis and renal transplant patients in a single-center study. Methods: The ioPTH was measured in 57 dialysis patients (33 females and 24 males) and 18 renal transplant recipients ( 12 males and 6 females) who underwent PTX from 2005 to 2015 for refractory HPT. Near-total PTX was performed in 56 patients and total PTX with autotransplantation in 20 patients. The PTH monitoring included 3 samples: pre-intubation, 10and 20-min (pre-ioPTH, 10-ioPTH, and 20-ioPTH) post parathyroid gland excision. Patients were followed up for up to 5 years. Results: In the dialysis group, the median (25th-75th
\end{abstract}

๑) 2017 S. Karger AG, Basel percentile) pre-, 10-, and 20-iOPTH levels were 1,447 pg/mL (938-2,176), $143 \mathrm{pg} / \mathrm{mL}$ (78-244) and $112 \mathrm{pg} / \mathrm{mL}$ (59-153) respectively. In the renal transplant group, pre-, 10-, and 20-ioPTH levels were $273 \mathrm{pg} / \mathrm{mL}$ (180-403), $42 \mathrm{pg} / \mathrm{mL}$ (25$72)$, and $34 \mathrm{pg} / \mathrm{mL}(23-45)$ respectively. All patients in the transplant group had a functional kidney transplant at the time of PTX with a median serum creatinine of $1.3 \mathrm{mg} / \mathrm{dL}$ $(1.2-1.7)$ and estimated glomerular filtration rate of $55 \mathrm{~mL} /$ min (40-60). The median time between renal transplant and PTX surgeries was 22 months (7-81). The last median followup PTH level was $66 \mathrm{pg} / \mathrm{mL}$ (15-201) in the dialysis group and $54 \mathrm{pg} / \mathrm{mL}(17-72)$ in the transplant group $(p=0.438)$. The mean time for last PTH post-PTX was $2.3 \pm 2.0$ years. In both groups, there was no significant difference between 20-ioPTH and any-time post-PTX PTH levels ( $p=0.6$ and $p=$ $0.9)$. Nineteen patients $(25 \%)$ were readmitted within 90 days because of hypocalcemia. One patient in the dialysis group was readmitted for post-PTX hematoma evacuation. No patient required repeat PTX because of recurrent HPT that was refractory to medical therapy. Only one dialysis patient required repeat PTX because the first procedure failed. Conclusions: The 20-ioPTH is a good indicator of long-term PTH levels in dialysis and renal transplant patients. Hypocal-

\section{KARGER}

E-Mail karger@karger.com

www.karger.com/nef
Dr. Amr El-Husseini

Division of Nephrology, Bone and Mineral Metabolism, University of Kentucky 800 Rose Street, Room MN-560

Lexington, KY 40536-0298 (USA)

E-Mail amr.elhusseini.moh@uky.edu 
cemia is a common complication, particularly in dialysis patients, and it is the main reason for readmission after PTX. Hypoparathyroidism is a potential concern after PTX in dialysis patients.

(c) 2017 S. Karger AG, Basel

\section{Introduction}

Despite advances in the pharmacological treatment for hyperparathyroidism (HPT), there are still some patients who cannot afford, do not tolerate or comply with, or do not respond to medical management. In this subset of patients, parathyroidectomy (PTX) might be warranted [1]. Successful PTX is determined primarily by a rapid reduction in serum PTH levels and amelioration of HPT symptoms [2]. There is inconsistency among parathyroid surgeons for what defines a successful PTX for our patients with renal diseases. Quick intraoperative PTH (ioPTH) assays are widely used to guide adequate parathyroid tissue resection during the surgery. However, the role of ioPTH monitoring in PTX for patients with renal insufficiency with multiple glandular hyperplasia is less clear than that in patients with primary HPT with single-gland disease. Furthermore, there is no consensus about the value of ioPTH to predict long-term PTH levels after PTX.

In most PTX surgeries, the target is a $70-80 \%$ reduction at 10 - and 20-min from the pre-ioPTH level. These figures are derived from a series of patients with primary HPT, when the PTH levels should drop back to the normal range after the surgery. Traditionally surgeons had been using the same ioPTH monitoring and targets in patients with renal diseases with secondary and tertiary HPT; however, the target intact PTH (iPTH) levels for these patients are different.

Ohe et al. [3] reported that a decrease of $80 \%$ or more of ioPTH level $20 \mathrm{~min}$ after PTX compared to pre-ioPTH predicts a $100 \%$ cure rate in secondary and tertiary HPT. However, they defined cure as a condition where there was no need for another PTX even if patients left the hospital with severe hypoparathyroidism. Zhang et al. [4], defined successful PTX as iPTH $<300 \mathrm{pg} / \mathrm{mL}$ after PTX. Overall, the mean percentage reduction of serum iPTH at 10 and 20 min after PTX in the successful PTX group were 86.8 and $90.6 \%$ respectively. At the time of discharge, the mean iPTH level had reduced from an average of 1300$54.7 \mathrm{ng} / \mathrm{L}$ and it was $<75 \mathrm{ng} / \mathrm{L}$ in $>90 \%$ of patients over a 3 -year period regardless of the surgical technique used.

There is inconclusive data on the proper usage of ioPTH and its association with long-term PTH levels In this study, we review the importance of ioPTH and its association with the long-term IPTH measurements after PTX in dialysis and renal transplant patients.

\section{Patients and Methods}

This study included all dialysis and kidney transplant patients who had undergone PTX for secondary and tertiary HPT between March 2005 and March 2015. The study was conducted according to the Declaration of Helsinki, and the protocol was reviewed and approved by the Institutional Review Boards of the University of Kentucky. Patients were identified using ICD-9 and -10 codes for PTX and secondary or tertiary HPT. An extensive retrospective chart review was the key to data gathering. The operative reports were checked individually for surgery indication and the procedure was performed. Additionally, the patients' preoperative visit notes, discharge summaries, and subsequent hospital visit and clinic notes were reviewed. Hospital and outpatient electronic medical records were accessed using Sunrise Clinical Manager, Allscripts TouchWorks EHR, and Organ Transplant Tracking Record. Furthermore, follow-up data from dialysis centers' medical records were collected for each patient to obtain iPTH measurements and other relevant laboratory values. Study data were collected and managed using REDCap electronic data capture tools hosted at the University of Kentucky.

PTX was performed for refractory HPT in the dialysis group and for hypercalcemia with persistent HPT in the transplant group. All PTX surgeries were performed by the same surgeon in the same institution. A total of 76 PTX were performed in 75 patients. One dialysis patient had an unsuccessful PTX because an ectopic gland could not be rightly located, and the patient underwent a successful repeat PTX 6 months later. Near-total PTX was performed in 56 patients and total PTX with autotransplantation in 20 patients. The ioPTH was measured in 57 dialysis patients ( 33 females and 24 males) and 18 renal transplant recipients ( 12 males and 6 females). The dialysis patients were either on peritoneal dialysis or hemodialysis at the time of PTX. At the time of PTX, the mean duration of dialysis was $4.4 \pm 2.4$ years. All patients in the transplant group had functioning transplants at the time of surgery.

The mean parathyroid gland (PTG) weight was $3.6 \pm 2.6 \mathrm{~g}$. The ioPTH monitoring included 3 samples: pre-intubation, 10-, and 20-min (pre-ioPTH, 10-ioPTH, and 20-ioPTH) post PTG excision. Intraoperative whole PTH was monitored using the QuickIntraoperative Bio-Intact PTH assay (Nichols Institute Diagnostics, San Clemente, CA, USA). The results were obtained within 9-10 min and they were helpful in guiding the surgeon to determine whether an appropriate amount of PTG was excised. The postoperative PTH levels were always iPTH, measured by a standard chemiluminesence immunoassay (Elecsys ${ }^{\circledR}$ System, Roche Diagnostics, Indianapolis, IN, USA). Normal values range from 15 to $65 \mathrm{pg} / \mathrm{mL}$; intra- and inter-assay coefficient of variations were $<6$ and $<9 \%$, respectively $[5,6]$. The Elecsys assay for determining iPTH employs a sandwich test principle in which a biotinylated monoclonal antibody reacts with the N-terminal fragment (1-37) and a monoclonal antibody labeled with a ruthenium complex reacts with the C-terminal fragment (38-84). We also collected the results of the ionized and serum calcium, albumin, and phosphorus levels perioperatively and during the follow-up period. Patients 
Table 1. Demographics and biochemical parameters

\begin{tabular}{lccc}
\hline & Dialysis $(n=57)$ & Transplant $(n=18)$ & $p$ value \\
\hline Age, years & $44.3 \pm 14.6$ & $56.1 \pm 9.1$ & $<0.001$ \\
Gender, male/female & $24 / 33$ & $12 / 6$ & 0.108 \\
Race (White/African American, number and \%) & $37 / 19(65 / 33)$ & $17 / 1(94 / 6)$ & 0.021 \\
Pre-ioPTH & $1,447(938-2,176)$ & $273(180-403)$ & $<0.001$ \\
10-ioPTH & $143(78-244)$ & $42(25-72)$ & $<0.001$ \\
20-ioPTH & $112(59-153)$ & $34(23-45)$ & $<0.001$ \\
Serum albumin, g/L & $3.4 \pm 0.5$ & $3.7 \pm 0.4$ & $\mathrm{~ns}$ \\
Serum calcium, mg/dL & $9.8 \pm 0.9$ & $10.5 \pm 0.7$ & $<0.001$ \\
Ionized calcium, mmol/L & $4.7 \pm 0.6$ & $5.4 \pm 0.4$ & $<0.001$ \\
Serum phosphorus, mg/dL & $6.2 \pm 2.4$ & $2.8 \pm 0.6$ & $<0.001$ \\
\hline
\end{tabular}

Values are mean $\pm S D$, median (25th-75th percentile). ns, non significant.

were followed up for up to 5 years with a mean period of $2.3 \pm 2.0$ years.

The characteristics of the dialysis and transplant patients who underwent PTX were evaluated using descriptive statistics means and standard deviations or medians and 25th-75th percentiles for continuous variables, and counts and percentages for categorical variables. Differences between groups were compared using independent samples $t$ test or Wilcoxon signed-rank sum test for continuous variables and chi-squared test for categorical variables. The Kruskal-Wallis 1-way analysis of variance complemented by Dunn test was used for comparing more than 2 sets of data. The resulting $p$ values are of a descriptive nature without any confirmatory value. To improve the presentation of the observed data, graphical methods were applied. All statistical analyses were performed by using SPSS version 23 (SPSS, Inc., Chicago, IL, USA). $p<0.05$ was considered statistically significant.

\section{Results}

Our study included 75 patients who had PTX between March 2005 and March 2015. There were 39 females (52\%) and $36(48 \%)$ males. The mean age at the time of PTX was $47.1 \pm 14.4$ years. Seventy-one percent were White and 27\% were African American. The main causes of endstage renal disease (ESRD) were glomerular diseases (31\%), hypertension (28\%), tubulointerstitial diseases (18\%), diabetes mellitus (12\%), and polycystic kidney disease (10\%). A majority of dialysis patients were on hemodialysis (96\%), while only $4 \%$ were on peritoneal dialysis.

In the dialysis group, the median (25th-75th percentile) pre-, 10-, and 20-ioPTH levels were 1,447 pg/mL (9382,176), $143 \mathrm{pg} / \mathrm{mL}$ (78-244), and $112 \mathrm{pg} / \mathrm{mL}$ (59-153), respectively. In the renal transplant group, pre-, 10-, and 20 -ioPTH levels were $273 \mathrm{pg} / \mathrm{mL}$ (180-403), $42 \mathrm{pg} / \mathrm{mL}$ (25-72), and $34 \mathrm{pg} / \mathrm{mL}(23-45)$ respectively. Compared to the dialysis group, transplant patients had significantly lower median, pre-, 10 -, and 20-ioPTH levels ( $p<0.001$ for all). All patients in the transplant group had a functional kidney transplant at the time of surgery with a median serum creatinine of $1.3 \mathrm{mg} / \mathrm{dL}(1.2-1.7)$ and estimated glomerular filtration rate of $55 \mathrm{~mL} / \mathrm{min}(40-60)$. The median time between renal transplant and PTX surgeries was 22 months (7-81). Demographic and laboratory parameters for both dialysis and transplant groups are shown in Table 1.

Among the dialysis patients, $71 \%$ had persistent low PTH levels after PTX (iPTH stayed less than $150 \mathrm{pg} / \mathrm{mL}$ throughout the entire follow-up period). Among transplant patients, $78 \%$ had iPTH levels less than $65 \mathrm{pg} / \mathrm{mL}$ during the follow-up period. The median last follow-up PTH level was $66 \mathrm{pg} / \mathrm{mL}$ (15-201) in the dialysis group and $54 \mathrm{pg} / \mathrm{mL}(17-72)$ in the transplant group $(p=0.438)$. In both groups, there was no significant difference between 20-ioPTH and follow-up PTH measurements ( $p=$ 0.6 and $p=0.9)$. There was no significant difference in follow-up PTH levels between groups $(p=0.998)$. The pre-PTX and follow-up PTH levels in both groups are shown in Figure 1. There were no statistically significant differences in pre-, 10-, 20-ioPTH, and follow up iPTH levels between patients who had near-total PTX and patients who had total PTX with autotransplantation (Fig 2). The baseline and follow-up ionized and corrected serum calcium and phosphorus levels are shown in Figures 3-5. There was no acute decline in kidney function after PTX in any of the transplant patients and the serum creatinine stayed stable throughout 1 year of follow-up (Fig. 6).

The mean \pm SD hospital stay was $4.6 \pm 3.7$ days. Nineteen patients $(25 \%)$ were readmitted within 90 days because of hypocalcemia ( 16 out of 57 in the dialysis group and 3 out of 18 in the transplant group, $p=0.37$ ). One patient in the 
Fig. 1. The pre-PTX and follow-up PTH levels in dialysis and transplant groups. ** Dialysis compared to the transplant group, $p<0.001$.

Fig. 2. The pre-PTX and follow-up PTH levels in patients who had near-total PTX compared to patients who had total PTX with autotransplantation. No significant differences in PTH levels between the 2 groups.
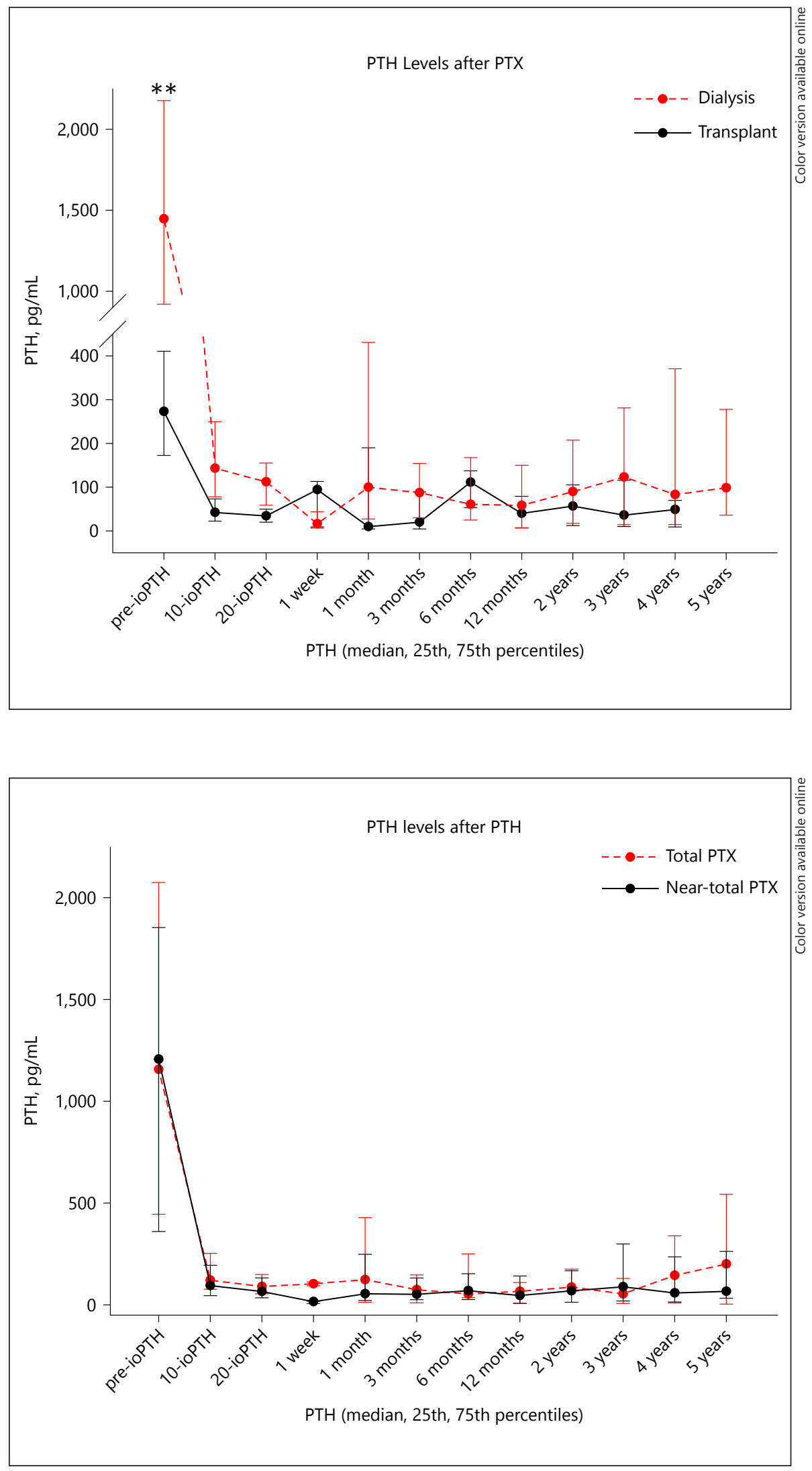
Fig. 3. Serum ionized calcium levels during PTX surgery. ${ }^{*}$ Transplant compared to dialysis group, $p<0.05$. ** Transplant compared to the dialysis group, $p<0.01$.
Fig. 4. Serum corrected calcium levels before and after PTX surgery. ${ }^{*}$ Transplant compared to the dialysis group, $p<0.05$. ** Transplant compared to the dialysis group, $p<0.01$.
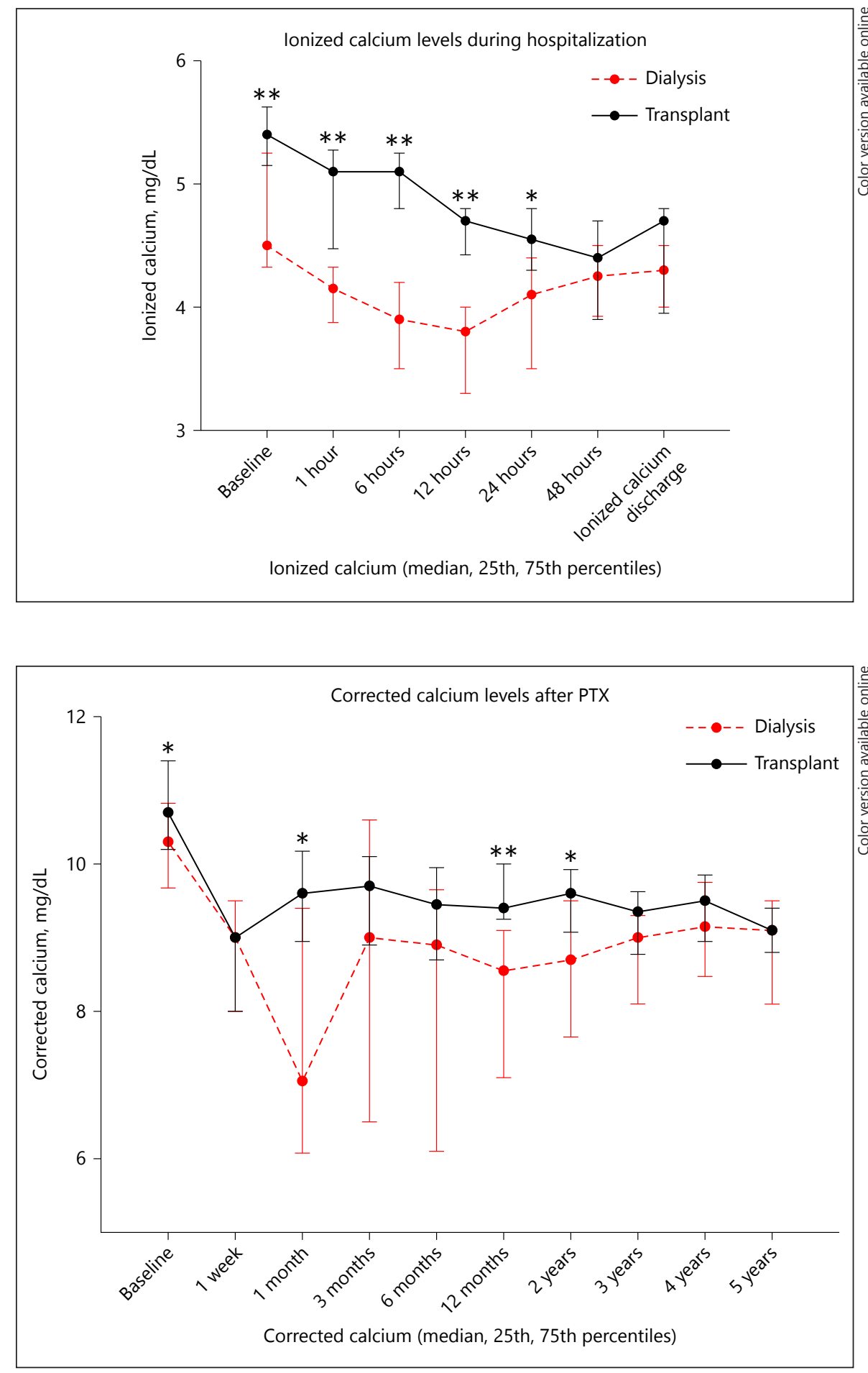

dialysis group was readmitted for post-PTX hematoma evacuation. No patient required repeat PTX because of recurrent HPT, but one patient in the dialysis group required repeat PTX because the first procedure failed. There were no postoperative mortalities among both groups.

\section{Discussion}

Advances in the medical intervention have limited the need for PTX in dialysis and renal transplant patients. Measuring $\mathrm{PTTH}$ is an established practice during PTX as 
Fig. 5. Serum phosphorus levels before and after PTX surgery. * Dialysis compared to the transplant group, $p<0.05 . * *$ Dialysis compared to the transplant group, $p<0.01$.

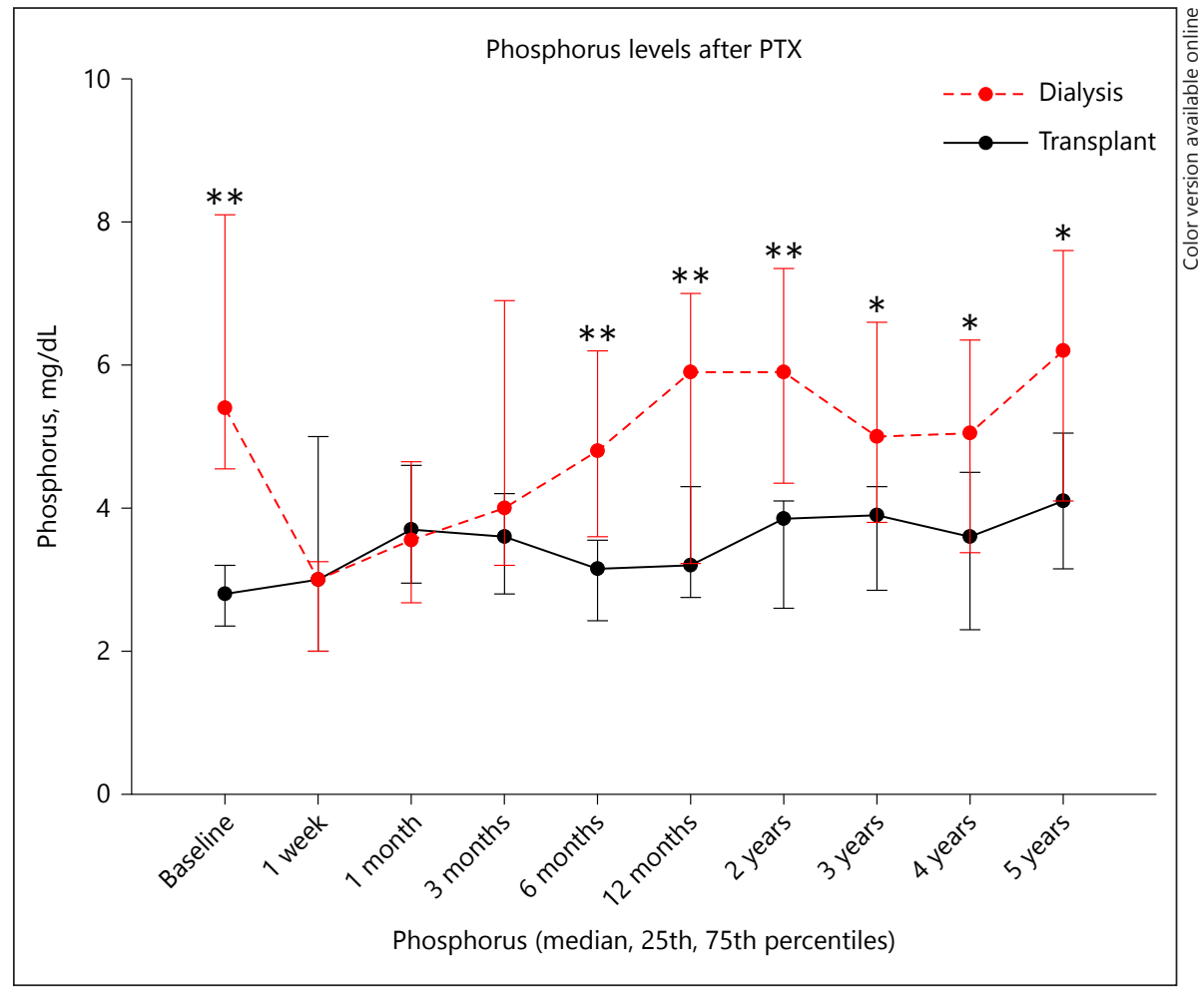

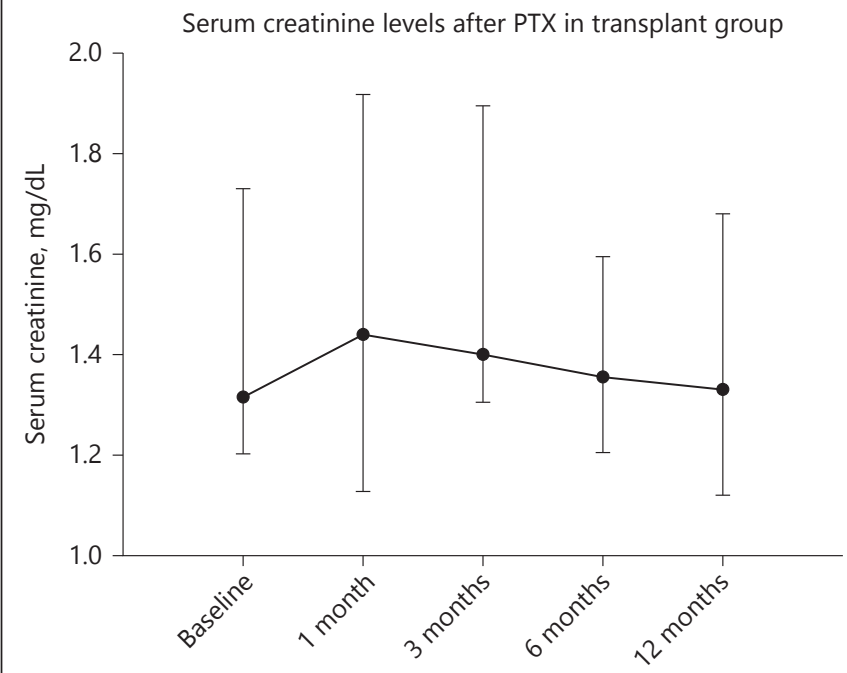

Fig. 6. Serum creatinine levels before and after PTX surgery in the transplant group. No significant change in serum creatinine after PTX. considerable number of patients have more than 4 PTG or ectopic glands $[5,6]$. Thus, if the sufficient removal of PTG can be intraoperatively confirmed, it may be possible to avoid further unnecessary explorations and prevent operative failure due to missed supernumerary glands. The ioPTH monitoring is a well-established modality for predicting surgical success in primary HPT [7-10], but its importance in secondary HPT is less well proven. The criteria of Quick-Intraoperative Bio-Intact PTH assay (Nichols Institute Diagnostics) for PTX of secondary HPT were well reported previously. The test was appropriate because it measured only whole PTH within 10-20 min. However, after the discontinuation of these assay kits, iPTH assay kits have now been widely used. To date, only a few studies have determined criteria to determine operative success rates using ioPTH monitoring for patients with secondary and tertiary HPT [11-16]. In this study, we aimed to retrospectively evaluate the association between ioPTH and long-term iPTH levels after PTX in dialysis and renal transplant patients. We found that the 20-ioPTH is a good indicator of long-term PTH levels in both groups. Damiano et al. compared the ioPTH and central laboratory PTH in 12 kidney transplants. They found excellent correlation between both assays and because of its accuracy it permits a reduction of operating 
time [11]. In a short-term study, Zhang et al. [4] evaluated the diagnostic accuracy of ioPTH in predicting successful PTX in 501 secondary HPT patients. In $89 \%$ or more of cases, ioPTH could predict successful PTX with a sensitivity rate of $78.6 \%$ and specificity rate of $88.5 \%$. In the STARD study, Hiramitsu et al. [12] and his group used $<60 \mathrm{pg} / \mathrm{mL}$ iPTH value on postoperative day one as an indicator of successful PTX in patients with secondary HPT. They found that a 70\% ioPTH drop from the baseline was appropriate to determine sufficient PTG removal during PTX. Ohe et al. [3] tried to define the ioPTH cutoff to predict successful PTX in secondary and tertiary HPT. A decrease of $80 \%$ or more in the ioPTH levels at 20 min predicted cure in all renal patients throughout the follow-up period. A decline of less than $70 \%$ predicted surgical failure in $66.6 \%$. A marginal ioPTH drop of 70 $79 \%$ was associated with $18.1 \%$ failure. On the other hand, Triponez et al. [13] studied the accuracy of ioPTH measurement during subtotal PTX for tertiary HPT after renal transplantation. The ioPTH had a high sensitivity. However, because of the low risk of persistent HPT, they concluded that its potential impact on the overall success rate is very less and they did not recommend the routine use of ioPTH in tertiary HPT. In a 1-year follow-up study, Conzo et al. [14] evaluated the role of ioPTH assay during PTX for secondary HPT. They reported that although the ioPTH assays are important, its predictive value was unsatisfactory. Moor et al. [15] examined the ioPTH monitoring to determine long-term success of total PTX for secondary HPT. They reported that ioPTH monitoring is of no use in predicting long-term cure for PTX because it does not predict success. Roshan et al. [16] examined the usefulness of ioPTH monitoring in secondary HPT and they concluded that the use of ioPTH monitoring failed to predict persistent hypoparathyroidism following total PTX but does predict biochemical resolution of HPT.

In our transplant patients, there was no acute decline in kidney function after PTX in any of them throughout the 1-year follow-up period. Chudzinski et al. [17] tried to answer the question: does PTX endanger the transplanted kidney? They found that PTX did not significantly impair the transplanted kidney function; however, transient reductions in graft function occurred in the early postoperative period. Meng et al. [18] recently reported on the safety aspects of PTX for persistent posttransplantation HPT. They reported similar pattern of acute graft deterioration after PTX that reversed to approximately pre-surgery levels 1 year after PTX. They did not report any episodes of intraoperative hypotension during the surgery. Thus, they explained this transient deterioration

Value of ioPTH during

Parathyroidectomy in Renal Patients of kidney function by the reversal of the hemodynamic effects of PTH as it exerts a significant afferent vasodilatory as well as efferent vasoconstrictory effects [19]. This finding has also been reported by others, but their results pointed to the long-term stability of the kidney function [20-23].

We did not find a significant difference between follow-up iPTH levels in dialysis and renal transplant groups. Also, the last iPTH levels were comparable in both groups (median iPTH was 54 in the renal transplant group versus $66 \mathrm{pg} / \mathrm{mL}$ in the dialysis group). All patients in the transplant group had functional allografts at the time of PTX with a median serum creatinine level of $1.3 \mathrm{mg} / \mathrm{dL}$ and estimated glomerular filtration rate of $55 \mathrm{~mL} / \mathrm{min}$, so iPTH levels were appropriate for the transplant group. However, in the dialysis group, the follow-up iPTH levels were much lower compared to the levels recommended by the National Kidney Foundation's Kidney Disease Outcomes Quality Initiative, and Kidney Disease: Improving Global Outcomes targets $[24,25]$. Moor et al. [15] showed that after PTX, patients are required to have long-term calcium and PTH assay follow-up because of tenacious hypoparathyroidism, as normoparathyroidism cannot be achieved. Zhang et al. [4] defined successful PTX as iPTH $<300 \mathrm{pg} / \mathrm{mL}$ after PTX. Overall, the mean percentage reduction of serum iPTH at 10 and $20 \mathrm{~min}$ after PTX in the successful PTX group were 86.8 and $90.6 \%$, respectively. Interestingly, many of their patients had very low and some of them had undetectable iPTH levels. Schlosser et al. [26] performed a pilot trial on 100 long-term dialysis patients with uncontrollable HPT with a 3-year follow-up period to compare total PTX with or without autotransplantation. At the time of discharge, the mean iPTH level fell from an average value of 1,300-54.7 $\mathrm{ng} / \mathrm{L}$ and it was $<75 \mathrm{ng} / \mathrm{L}$ in $>90 \%$ of patients over a 3 -year period regardless of the surgical technique used.

In this study, the mean hospital stay was 4.6 days; this was similar to the average US national PTX hospital stay for hemodialysis patients with HPT, as Ishani et al. [27] reported a 4-days index hospitalization (25th/75th percentile, 3-7 days) in a nationwide database. Hypocalcemia was a common complication and the main reason for readmission after PTX in our patients (one-quarter of patients were readmitted within 3 months because of hypocalcemia). We did not report any early postoperative serious cardiac complications or mortality. No patient required repeat PTX because of recurrent HPT, but one patient required repeat PTX because the first procedure failed and one patient was readmitted for post-PTX hematoma evacuation. PTX is associated with a low rate of 
recurrent HPT. However, the clear disadvantage is permanent hypoparathyroidism, and patients undergoing PTX require lifelong calcium and vitamin D replacement. The early postoperative morbidity and mortality of PTX was not trivial after PTX in previous studies. Analysis of large national data for dialysis patients showed a mortality rate of $2 \%$ in the first month after PTX [27]. During the 30 days after discharge, $24 \%$ of patients were rehospitalized; $29 \%$ of these patients required intensive care treatment. Compared to the year prior to PTX, in the year following the surgery, hospitalizations were higher by $39 \%$, hospital days by $58 \%$, intensive care unit admissions by $69 \%$, and there was a 20 -fold increase in emergency room/ observation visits requiring hypocalcemia treatment. Cause-specific hospitalizations were higher for acute myocardial infarction and dysrhythmia [27]. It seems as if hypoparathyroidism develops regardless of the surgical technique used and ESRD patients are at risk for permanent hypoparathyroidism after any type of PTX. We did not find any statistically significant difference between 10-, 20-ioPTH, and follow-up iPTH levels in patients who had near-total PTX compared to patients who had total PTX with autotransplantation. This was also reported in a prospective multicenter randomized controlled pilot trial [26].

Hypoparathyroidism was a major concern after PTX for our dialysis patients. The impact of low PTH levels after PTX on bone and patient survivals was not explored judiciously in prospective studies and was beyond the scope of our study. However, hypoparathyroidism is a potential cause of low bone turnover disease, which is associated with bone fractures and cardiovascular morbidity and mortality. Hernandes et al. [28] studied the effect of PTX on bone turnover and cardiovascular calcification in 19 dialysis patients. At the end of one year, $90 \%$ of the patients evolved from high to low turnover bone disease and calcium scores increased significantly; there was a positive association between vascular calcification progression and the severity of low turnover bone disease. The shift from high to low bone turnover disease after PTX might be causing progression of vascular calcification and could be associated with increased patient mortality.

Nephrologists are generally very familiar with the treatment of HPT and have several therapeutic options. This is not the case when it comes to hypoparathyroidism. Parathyroid cryopreservation has been advocated for many years to enable delayed heterotopic parathyroid autotransplantation for hypoparathyroid patients, but it is costly, time consuming, and therefore never used in clinical practice [29]. We previously reported that the def- inition of successful PTX in dialysis patients needs to be revised to include not only a minimal but also a maximal decrease of PTX levels. Targeting postoperative PTH levels of at least $150 \mathrm{pg} / \mathrm{mL}$ seems reasonable for our dialysis patients [1]. Also based on the available literature, using an arbitrary definition of a decrease of at least $80 \%$ of the preoperative PTH level may be too ample, and instead of using percentage reduction we can maintain postoperative PTH levels as recommended by Kidney Disease Outcomes Quality Initiative and Kidney Disease: Improving Global Outcomes for dialysis patients.

Despite a better biochemical control of SHP, no solid evidence has been produced demonstrating a positive impact of PTX on mortality or other surrogate outcomes. Ivarsson et al. [30] studied the effect of PTX on patient survival using the Swedish Renal Registry. Incident dialysis patients with PTX $(n=423)$ were compared with nonPTX patients $(n=1,234)$ and matched for gender, age, cause of ESRD. They concluded that PTX was associated with improved survival (HR 0.80). These results are consistent with other observational studies that were also based on large national registry with a long follow-up [3134]. In an editorial, however, Messa questioned the better outcomes of PTX cohorts because of a major problem with the studies that defined the indications for the intervention and selection of surgical candidates [35]. Further clouding the reported benefits of PTX are other studies that show no survival benefits of PTX in this population [36, 37]. Komaba et al. [32] studied the survival patterns among Japanese hemodialysis patients using the propensity score to match PTX and non-PTX patients. The authors reported that PTX patients had significantly lower risks of 1-year all-cause and cardiovascular mortalities. They concluded that successful PTX may reduce the risk for all-cause and cardiovascular mortalities in hemodialysis patients with severe, uncontrolled HPT. Julia Scialla and Myles Wolf commented that despite the strengths of this study, it has several limitations related to its unorthodox design. It was not an ideal observational study where clinical data are collected during the pre-PTX period; the start of survival follow-up is linked to the time when the indication for PTX is first established; and all subsequent deaths are captured [38]. The only definitive strategy to eliminate confounding by indication is randomization, but when it comes to PTX in dialysis patients, this is unlikely to happen. Chen et al. [39] published a systematic review and meta-analysis on long-term mortality after PTX among CKD patients with secondary HPT. They included 13 cohort studies involving 22,053 patients in their analysis. They found PTX-treated versus medically treat- 
ed patients had a $28 \%$ reduction in all-cause mortality and a $37 \%$ reduction in cardiovascular mortality. They concluded that PTX might reduce the risks of all-cause and cardiovascular mortalities in CKD patients with HPT. However, once again a critical and unsolved question is related to how correctly the control group for the PTX cohorts can be defined. All the reported studies are potentially biased in that the patients who are deemed ineligible for PTX might be at higher mortality risk than those in the PTX cohort. A more definite conclusion about the mortality effects of PTX could be reached if a prospective controlled trial was directed at mitigating this control group problem.

\section{Conclusions}

The 20-ioPTH is a good indicator of long-term PTH measurements in dialysis and renal transplant patients. Hypocalcemia is a common complication and the main reason for readmission after PTX. Hypoparathyroidism is a major concern after PTX for dialysis patients.

\section{Acknowledgments}

The project described is supported by the NIH National Center for Advancing Translational Sciences through grant number UL1TR001998. The content is solely the responsibility of the authors and does not necessarily represent the official views of the NIH.

\section{Ethics Statement}

This study did not require informed consent nor review/approval by the appropriate ethics committee.

\section{Disclosure Statement}

The authors have no conflicts of interest to declare.

\section{References}

1 El-Husseini A, Wang K, Edon AA, Sawaya BP: Parathyroidectomy-A Last Resort for Hyperparathyroidism in Dialysis patients. Semin Dial 2017;30:385-389.

2 Cozzolino M, Elli F, Carugo S, Ciceri P: Secondary hyperparathyroidism in end-stage renal disease: no longer a matter for surgeons? Blood Purif 2016;42:44-48.

3 Ohe MN, Santos RO, Kunii IS, Carvalho AB, Abrahão $\mathrm{M}$, Neves MC, Lazaretti-Castro $\mathrm{M}$, Cervantes O, Vieira JG: Intraoperative PTH cutoff definition to predict successful parathyroidectomy in secondary and tertiary hyperparathyroidism. Braz J Otorhinolaryngol 2013;79:494-499.

4 Zhang L, Xing C, Shen C, Zeng M, Yang G, Mao H, Zhang B, Yu X, Cui Y, Sun B, Ouyang C, Ge Y, Jiang Y, Yin C, Zha X, Wang N: Diagnostic accuracy study of intraoperative and perioperative serum intact PTH level for successful parathyroidectomy in 501 secondary hyperparathyroidism patients. Sci Rep 2016; 27:26841.

5 Akerström G, Malmaeus J, Bergström R: Surgical anatomy of human parathyroid glands. Surgery 1985;95:14-21.

6 Phitayakorn R, McHenry CR: Incidence and location of ectopic abnormal parathyroid glands. Am J Surg 2006;191:418-423.

7 Nussbaum SR, Thompson AR, Hutcheson KA, Gaz RD, Wang CA: Intraoperative measurement of parathyroid hormone in the surgical management of hyperparathyroidism. Surgery 1988;104:1121-1127.

8 Bergenfelz A, Isaksson A, Lindblom P, Westerdahl J, Tibblin S: Measurement of parathy- roid hormone in patients with primary hyperparathyroidism undergoing first and reoperative surgery. Br J Surg 1998;85:1129-1132.

9 Westerdahl J, Lindblom P, Bergenfelz A: Measurement of intraoperative parathyroid hormone predicts long-term operative success. Arch Surg 2002;137:186-190.

10 Ohe MN, Santos RO, Kunii IS, Carvalho AB, Abrahão $\mathrm{M}$, Cervantes $\mathrm{O}$, et al: Usefulness of a rapid immunometric assay for intraoperative parathyroid hormone measurements. Braz J Med Biol Res 2003;36:715-721.

11 Damiano G, Gioviale MC, Maione C, Sacco M, Buscemi S, Palumbo VD, Spinelli G, Ficarella S, De Luca S, Maffongelli A, Fazzotta S, Carmina L, Buscemi G, Lo Monte AI: Comparison between rapid intraoperative and central laboratory parathormone dosage in 12 kidney transplant candidates. Transplant Proc 2016;48:311-314.

12 Hiramitsu T, Tominaga Y, Okada M, Yamamoto T, Kobayashi T: A retrospective study of the impact of intraoperative intact parathyroid hormone monitoring during total parathyroidectomy for secondary hyperparathyroidism: STARD study. Medicine (Baltimore) 2015;94:e1213.

13 Triponez F, Dosseh D, Hazzan M, Noel C, Soudan B, Lokey J, Mozzon M, Proye CA: Accuracy of intra-operative PTH measurement during subtotal parathyroidectomy for tertiary hyperparathyroidism after renal transplantation. Langenbecks Arch Surg 2006;391: 561-565.

14 Conzo G, Perna A, Avenia N, De Santo RM, Della Pietra C, Palazzo A, Sinisi AA, Stanzi- one F, Santini L: Evaluation of the 'putative' role of intraoperative intact parathyroid hormone assay during parathyroidectomy for secondary hyperparathyroidism. A retrospective study on 35 consecutive patients. Endocrine 2012;42:606-611.

15 Moor JW, Roberts S, Atkin SL, England RJ: Intraoperative parathyroid hormone monitoring to determine long-term success of total parathyroidectomy for secondary hyperparathyroidism. Head Neck 2011;33:293296.

16 Roshan A, Kamath B, Roberts S, Atkin SL, England RJ: Intra-operative parathyroid hormone monitoring in secondary hyperparathyroidism: is it useful? Clin Otolaryngol 2006;31:198-203.

17 Chudzinski W, Wyrzykowska M, Nazarewski S, Durlik M, Galazka Z: Does the parathyroidectomy endanger the transplanted kidney? Transplant Proc 2016;48:1633-1636.

18 Meng C, Martins P, Frazão J, Pestana M: Parathyroidectomy in persistent post-transplantation hyperparathyroidism-single-center experience. Transplant Proc 2017;49:795798.

19 Massfelder T, Parekh N, Endlich K, Saussine C, Steinhausen M, Helwig JJ: Effect of intrarenally infused parathyroid hormone-related protein on renal blood flow and glomerular filtration rate in the anaesthetized rat. $\mathrm{Br} \mathrm{J}$ Pharmacol 1996;118:1995-2000.

20 Garcia A, Mazuecos A, Garcia T, González P, Ceballos M, Rivero M: Effect of parathyroidectomy on renal graft function. Transplant Proc 2005;37:1459-1461.
Value of ioPTH during

Parathyroidectomy in Renal Patients
Nephron 2018;138:119-128 DOI: $10.1159 / 000482016$ 
21 Ferreira GF, Montenegro FL, Machado DJ, Ianhez LE, Nahas WC, David-Neto E: Parathyroidectomy after kidney transplantation: short-and long-term impact on renal function. Clinics (Sao Paulo) 2011;66:431-435.

22 Evenepoel P, Claes K, Kuypers D, Maes B, Vanrenterghem Y: Impact of parathyroidectomy on renal graft function, blood pressure and serum lipids in kidney transplant recipients: a single centre study. Nephrol Dial Transplant 2005;20:1714-1720.

23 Kandil E, Florman S, Alabbas H, Abdullah O, McGee J, Noureldine S, Slakey D, Zhang R: Exploring the effect of parathyroidectomy for tertiary hyperparathyroidism after kidney transplantation. Am J Med Sci 2010;339:420424.

24 National Kidney Foundation: K/DOQI clinical practice guidelines for bone metabolism and disease in chronic kidney disease. Am J Kidney Dis 2003;42(4 suppl 3):S1-S201.

25 Kidney Disease: Improving Global Outcomes (KDIGO) CKD-MBD Work Group: KDIGO clinical practice guideline for the diagnosis, evaluation, prevention, and treatment of chronic kidney disease-mineral and bone disorder (CKD-MBD). Kidney Int Suppl 2009; 113:S1-S130.

26 Schlosser K, Bartsch DK, Diener MK, Seiler CM, Bruckner T, Nies C, Meyer M, Neudecker J, Goretzki PE, Glockzin G, Konopke R, Rothmund M: Total parathyroidectomy with routine thymectomy and autotransplantation versus total parathyroidectomy alone for secondary hyperparathyroidism: results of a nonconfirmatory multicenter prospective randomized controlled pilot trial. Ann Surg 2016;264:745-753.

27 Ishani A, Liu J, Wetmore JB, et al: Clinical outcomes after parathyroidectomy in a nationwide cohort of patients on hemodialysis. Clin J Am Soc Nephrol 2015;10:90-97.

28 Hernandes FR, Canziani ME, Barreto FC, et al: The shift from high to low turnover bone disease after parathyroidectomy is associated with the progression of vascular calcification in hemodialysis patients: a 12-month followup study. PLoS One 2017;12:e0174811.

29 Shepet K, Alhefdhi A, Usedom R, Sippel R, Chen H: Parathyroid cryopreservation after parathyroidectomy: a worthwhile practice? Ann Surg Oncol 2013;20:2256-2260.

30 Ivarsson KM, Akaberi S, Isaksson E, et al: The effect of parathyroidectomy on patient survival in secondary hyperparathyroidism. Nephrol Dial Transplant 2015;30:20272033.

31 Kestenbaum B, Andress DL, Schwartz S, et al: Survival following parathyroidectomy among United States dialysis patients. Kidney Int 2004;66:2010-2016.

32 Komaba H, Taniguchi M, Wada A, Iseki K, Tsubakihara Y, Fukagawa M: Parathyroidectomy and survival among Japanese hemodialysis patients with secondary hyperparathyroidism. Kidney Int 2015;88:350-359.
33 Jean G, Souberbielle JC, Zaoui E, et al: Analysis of the kinetics of the parathyroid hormone, and of associated patient outcomes, in a cohort of haemodialysis patients. BMC Nephrology 2016;17:153.

34 Chen JB, Chou FF, Yang CH, Hua MS: Association between clinical variables and mortality after parathyroidectomy in maintenance hemodialysis patients. Am J Surg 2017;213: 140-145.

35 Messa P: Parathyroidectomy and patient survival in CKD patients. Nephrol Dial Transplant 2015;30:1944-1946.

36 Trombetti A, Stoermann C, Robert J, et al: Survival after parathyroidectomy in patients with end-stage renal disease and severe hyperparathyroidism. World J Surg 2007;31: 1014-1021.

37 Conzo G, Perna AF, Savica V, et al: Impact of parathyroidectomy on cardiovascular outcomes and survival in chronic hemodialysis patients with secondary hyperparathyroidism. A retrospective study of 50 cases prior to the calcimimetics era. BMC Surgery 2013; 13(suppl 2):S4.

38 Scialla JJ, Wolf M: When there will never be a randomized controlled trial. Kidney Int 2015; 88:220-222.

39 Chen L, Wang K, Yu S, et al: Long-term mortality after parathyroidectomy among chronic kidney disease patients with secondary hyperparathyroidism: a systematic review and meta-analysis. Ren Fail 2016;38:1050-1058. 